\title{
Diagnosis associated with COVID-19 (U07.1), Pneumonia- Unspecified (J18.9) and other types of admissions: A restricted medical scheme case study- South Africa
}

\author{
Michael Mncedisi Willie ${ }^{1^{*}}$, Asnath Motsepe ${ }^{2}$ \\ ${ }^{1}$ Policy Research and Monitoring, Council for Medical Schemes, Pretoria, South Africa, \\ ${ }^{2}$ Sefako Makgatho Health Sciences University, Ga-Rankuwa, Pretoria, South Africa
}

\section{Accepted $30^{\text {th }}$ January, 2021}

The primary objective of this study was to assess the characteristics of patients admitted for COVID-19, 'J18.9 Pneumonia, unspecified organism' and other types of diagnoses. The aim was to assess as to what extent do COVID-19 related admissions changed to pneumonia, and as to what extent do 'J18.9 Pneumonia, unspecified organism' related admissions that changed to COVID-19 diagnosis at discharge stage. The secondary objective of the study was to assess' predictors of readmissions in private hospitals. The review period was private hospital claims received by the scheme between January and August 2020. The inclusion criteria for COVID-19 admissions were patients that had a laboratory-confirmed (RT - PCR assay) COVID-19. Predictors of readmissions were modelled using logistic regression. The study found that restricted scheme patients admitted for a COVID-19 diagnosis changed to a 'J18.9 Pneumonia, unspecified organism' diagnosis. The converse was found to be true in that some patients that were admitted as J18.9 Pneumonia, unspecified organism' diagnosis changed to a COVID-19 diagnosis. This study showed underlying factors associated with hospital admissions and predictors of readmissions in private hospitals.

Keywords: COVID-19, Diagnosis, Hospitalisation, ICD-10, Pneumonia, Risk factors

\section{INTRODUCTION}

The COVID-19 pandemic has adversely affected socio-economic and health systems globally. The absolute speed of both the geographical growth and the sudden increase in numbers of new cases startled and rapidly overwhelmed public and private health services in South Africa (SA) (Wu and McGoogan, 2020). Most health systems worldwide are overwhelmed because of the unprecedented spread of the virus. Previous pandemics have had persistent mental health effects. 'J18.9 Pneumonia, unspecified organism'

*Corresponding Author Email:

m.willie@medicalschemes.co.za is amongst the top five most prevalent reasons for admissions in medical schemes (CMS, 2019). This further accounts for a significant proportion of hospitalisation expenditure.

The origin of the virus

A series of 'J18.9 Pneumonia, unspecified organism' cases of unknown cause emerged in Wuhan, Hubei, China, with clinical presentations much similar to viral pneumonia. Analysis from lower respiratory tract samples indicated a novel coronavirus, which was named 2019 novel coronavirus (2019-nCoV) (Huang et al., 2020). 
The first outbreak of coronavirus disease (COVID19) happened in Wuhan, China, in December 2019 (Jager et al., 2020). Since then, the virus has spread rapidly across the world, prompting a global pandemic. The COVID-19 is caused by severe acute respiratory syndrome coronavirus 2 (SARS-CoV-2) virus which causes pneumonia. However, in more severe cases, it affects other organs (Jager et al., 2020). The occurrence of severe acute respiratory syndrome coronavirus 2 (SARS-CoV-2), which causes coronavirus disease (COVID-19), has presented a daunting medical challenge to health systems and clinicians globally (Garcia-Vidal et al., 2020).

The most common symptoms of COVID-19 are fever, tiredness, and dry cough. Some patients may have aches and pains, nasal congestion, runny nose, or sore throat. These symptoms are usually mild and begin gradually. Most people (about $80 \%$ ) recover from the disease without needing special treatment, and for the majority, children, and young adults, illness due to COVID19 is generally minor. However, for some people, it can cause serious illness. People who are over 60 years of age and people who have underlying medical conditions such as diabetes, heart disease, respiratory disease, or hypertension are among those who are at greater risk of developing severe or critical illness should they be infected with the virus. (WHO Media Statement: Knowing the risks for COVID-19, 2020).

\section{Complications, risk factors and mortality rate of COVID-19}

It is noted that COVID-19 can prompt severe lung injury leading to acute respiratory distress syndrome (ARDS), particularly in older patients and those with comorbidities. Patients suffering from interstitial lung diseases (ILDs) could also be at an increased risk of experiencing a rapid eruption of the disease due to COVID-19. However, this hypothesis is based on causal diseases and the median age of a population, which is commonly above 60 years (Guiot et al., 2020). The clinical spectrum of COVID-19 contrasts widely, from asymptomatic disease to 'J18.9 Pneumonia, unspecified organism' and lifethreatening complications, which includes acute respiratory distress syndrome (ARDS), multisystem organ failure, and ultimately death
(Berenguer et al., 2020). While men and women have the same prevalence of COVID-19, men with COVID-19 are more at risk for worse outcomes and death, independent of age (Jin et al., 2020). Early identification of risk factors for critical conditions is urgently needed, not only to identify the defining clinical and epidemiological characteristics with greater accuracy but also to simplify the appropriate supportive care and prompt access to the intensive care unit (ICU) if required (Jin et al., 2020). According to Himmels et al. (2020), increasing age was found to be the strongest conjecturer of death due to COVID-19 complications. Male gender, number of and severity of most comorbidities were found to be significant predictors of COVID-19-related death. Ethnicity other than white was consistently associated with modest but increased risk of death, with Blacks having the highest risk of death. A study by Himmels et al. (2020) found an increased risk of death with an increasing degree of deprivation. Other comorbidities such as chronic renal failure or kidney disease, diabetes mellitus type I and II, dementia, chemotherapy, organ transplant patients, severe immunodeficiency, cerebral palsy, and Down syndrome were reported as the strongest predictors of COVID-19 related death (OR/HR $>=3$ ) (Himmels et al., 2020).

\section{Association of 'J18.9 Pneumonia, unspecified organism' with COVID-19}

According to the Centre for Disease Control and Prevention (CDC), in the United States, the common causes of viral 'J18.9 Pneumonia, unspecified organism' are influenza, respiratory syncytial virus (RSV), and SARS-CoV-2 (the virus that causes COVID-19). A common cause of bacterial 'J18.9 Pneumonia, unspecified organism' is Streptococcus pneumonia (pneumococcus). (Causes of 'J18.9 Pneumonia, unspecified organism' CDC, 2020) Because 'J18.9 Pneumonia, unspecified organism' caused by SARS-CoV-2 is similar to idiopathic interstitial 'J18.9 Pneumonia, unspecified organism' (IIP), as a result, this often makes the diagnosis difficult (Fujita, 2020).

In terms of section 67 of the Medical Schemes Act, the Minister of Health published an amendment to the regulations in Notice 515 in Government Gazette 43295 (CMS, 2020); after a 
submission by the CMS to include COVID-19 as a Prescribed Minimum Benefit (PMB). COVID-19 infection may result in various complications, and most of the complications are included in the PMBs and should be treated as specified for the specific condition. One of the most common complications of COVID-19 infection, 'J18.9 Pneumonia, unspecified organism' is a PMB condition under the diagnosis and treatment pairs (DTP) code 903D. This DTP refers to "bacterial, viral, fungal pneumonia." The treatment component for this condition is specified as "Medical Management, Ventilation."

All medical schemes are required by law to pay for the diagnosis, treatment \& care costs for this condition in full, irrespective of plan type or option. Medical schemes are not allowed to fund PMB conditions from a member's medical scheme savings account, as this is not in line with the $\mathrm{PMB}$ regulations.

In a study conducted by Heugel, Martin, Kuypers, and Englund in 2007, it was found that coronavirus might also play a pathogenic role either on its own or possibly with a bacterial pathogen in the lower respiratory tract disease among children without the primary cardiopulmonary disease, immunosuppression, or a history of prematurity. The clinical cases they reported on provided a clinical description of radiographically confirmed coronavirus-associated pneumonia in previously healthy children. Additionally, another case demonstrated that coronavirus-associated pneumonia could have substantial clinical outcomes, resulting in infant intensive care unit admissions even among children without chronic primary health issues (Heugel, Martin, Kuypers, and Englund, 2007).

\section{Hospital length of stay for 'J18.9 Pneumonia, unspecified organism' with and without COVID 19}

According to the report by the American Journal of Managed Care, patients who presented to the hospital with pneumonia, intensive care unit stays increased their time spent in the hospital by 50 percent, bringing their length of stay to 7.2 days (Bai, 2018). The report from the National Hospital Care Survey (2014) only included patients who were hospitalised primarily for 'J18.9 Pneumonia, unspecified organism' and there were three report insights, which stated the following; 'J18.9 Pneumonia, unspecified organism' hospitalisations made up 2.1 percent of all discharges, with 46.7 percent of hospitalisations happening in patients 65 years and older; visits to the emergency department due to 'J18.9 Pneumonia, unspecified organism' were 0.5 percent of all emergency department visits, with 46.1 percent of them happening in patients under 15 years old. The study found the average length of stay in the hospital was 4.2 days for pneumonia, which is not associated with COVID19. The shortest length of stay was 3.1 days for patients under 15 years old, and the longest length of stay of 4.6 days for patients 65 years and older (Knowles, 2020).

Contrary to this, since the first identified case of COVID-19 in South Africa (SA) on 5 March 2020, the number of confirmed cases has rapidly increased, and as of 20 September 2020 amounted to 659656 (Audley et al., 2020). One in five people with confirmed COVID-19 requires hospitalisation. In most cases, the indication for hospitalisation is hypoxemia requiring supplemental oxygen therapy, the duration of which ranges from days to months, putting tremendous strain on hospital infrastructure, particularly central oxygen supply. Patients hospitalised with COVID-19 have varying levels of supplemental oxygen requirements (Audley et al., 2020)..

\section{Causes/ risk factors of readmissions in patients with COVID 19}

According to Rokadiya, Gil, Stubbs, Bell \& Herbert, R. (2020), clinical outcomes from hospitalised patients with COVID-19 are poor, with a reported mortality rate of $21 \%$. Age, sex, comorbidities, ethnicity, and deprivation, have all been shown to correlate with worse outcomes in patients with COVID-19; however, the outcomes of hospitalised patients once discharged remains unknown. (Rokadiya S., Gil E., Stubbs C., Bell D., Herbert R. 2020), The authors conducted a study where these outcomes were investigated in all patients with COVID-19, once discharged, were referred to the Home Referral Team (HRT), a team of clinicians specialising in General Practice who would follow up patients with a phone call 
either daily or every three days depending on COVID severity unless discharged to a care home or another treatment centre.

Demographic, clinical, biochemical, and radiological metadata were retrospectively collected and interrogated for all medical patients with confirmed SARS-CoV-2 infection between 09/03/2020 and 30/04/2020. SARS-CoV-2 PCR tests were performed on 1911 patients, 729 of whom (38\%) tested positive, 391 of whom were discharged. Out of these patients, 39 patients returned to the hospital emergency department, with 25 of them requiring readmission. Readmitted patients were further divided into those with dyspnoea as their primary complaint. The median age of the re-admitted group was higher at 73 years (IQR 58-82) compared to discharged patients of 59 years (IQR 48-76). Sex distribution was $60 \%$ male $(15 / 25)$ in the readmission group versus $58.8 \%$ female (429/729) in all COVID-19 patients, and ethnicity was predominantly of Black, Asian, or Minority Ethnic (BAME) background in both groups with absolute $62.5 \%$ discharged vs. $57.1 \%$ readmission. Biomarker results were similar in both groups. Of those readmitted; two patients required Intensive Care management, three patients died, and three further patients were discharged home as part of their end-of-life care. (Rokadiya S., Gil E., Stubbs C., Bell D., Herbert R., 2020). This highlights some of the risk factors of readmissions among patients with COVID 19 who have been discharged.

\section{Previous studies}

A study by (Chen et al., 2020) to clarify the epidemiological and clinical characteristics of 2019-nCoV pneumonia, it was found that, of the 99 patients with 2019-nCoV pneumonia. The average age of the patients was older than 50 years at 55.5 years (SD 13.1), the patients mainly included males than females, 67 and 32 women, respectively. Patients with clinical manifestations of fever were $83 \%$, the cough was $82 \%$, shortness of breath was $31 \%$, muscle ache were $11 \%$, confusion was $9 \%$, the headache was $8 \%$, sore throat was $5 \%$, rhinorrhea were $4 \%$, chest pain was $2 \%$, diarrhoea was $2 \%$, and nausea and vomiting were $1 \%$. Based on radiographic imaging Examination, $75 \%$ of patients showed bilateral pneumonia, $14 \%$ of patients showed multiple mottling and ground-glass opacity, and $1 \%$ of patient had a pneumothorax.

Another study conducted by (Garcia-Vidal et al., 2020) assessed 989 consecutive adults with COVID-19 who either had been discharged or died during the review period. The study mainly included males, 552 (55.8\%) were male; the median (IQR) age was 62 years.

\section{Legislative requirement}

The Medical Schemes Act No 131 of 1998 makes it mandatory for medical schemes to cover costs for the diagnosis, treatment, and care of a defined set of benefits (Willie, 2020). The prescribed minimum benefits (PMBs), regardless of the benefit option members have selected. PMBs include any medical condition that meets the definition of an emergency, a limited set of 270 medical conditions, and 26 chronic conditions, defined in the Chronic Disease List (CDL). The CDL specifies medication and treatment for the chronic conditions that are covered as PMBs. This law ensures that patients with chronic conditions are not risk-rated.

\section{'J18.9 Pneumonia, unspecified organism' and COVID-19 as Prescribe Minimum Benefit (PMB)}

The Ministry of Health, in terms of section 67 of the Medical Schemes Act, published an amendment to the regulations in Notice 515 in Government Gazette 43295 (CMS, 2020); after a submission by the CMS to include COVID-19 as a PMB. COVID-19 infection may result in various complications. Most of the complications are in any event included in the PMBs list of conditions and should be treated as specified for the specific condition. One of the most common complications of COVID-19 infection - 'J18.9 Pneumonia, unspecified organism' is a PMB level of care.

Medical schemes are required as per the Medical Schemes Act 131 of $1998^{i}$ (MSA) to pay for the diagnosis, treatment, and care for all PMB conditions and these must be covered in full, irrespective of plan type or option. Furthermore, medical schemes are not allowed to fund PMB conditions from the medical savings account. Funding PMBs from the medical savings account is prohibited as it is against the PMB regulations and Code of conduct (CMS, 2013). 


\section{Study objectives}

'J18.9 Pneumonia, unspecified organism' is among the top five reasons for admissions in medical schemes and among the most common conditions encountered worldwide. Studies have shown that chronic conditions such as hypertension and diabetes could significantly increase the risk of severity and fatality in COVID19 patients so as Pneumonia Clinical characteristics of COVID-19 related pneumonia patients have been shown in recent studies (Wang et al., 2020; Chen et at, 2020; Zhu et al., 2020). 'J18.9 Pneumonia, unspecified organism' is reported to be one of the complications of COVID-19. The current study assessed characteristics and risk factors of patients admitted for COVID-19 and 'J18.9 Pneumonia, unspecified organism' and other non-COVID-19 related conditions.

\section{METHODOLOGY}

\section{Study design}

The study design was a descriptive cross-section analysis of a restricted medical scheme'si" claims information. The review period was claims received between January and August 2020. Inclusion criteria for COVID-19 admissions were patients that had a laboratory-confirmed (RT PCR assay) COVID-19. The World Health Organization (WHO) defines ICD-10 codes 'U07.1' and 'U07.2' as follows (WHO, 2020):

An emergency ICD-10 code of 'U07.1 COVID-19, virus identified' is assigned to a diagnosis of COVID-19 confirmed by laboratory testing; An emergency ICD-10 code of 'U07.2 COVID-19, virus not identified' is assigned to a clinical or epidemiological diagnosis of COVID-19 where laboratory confirmation is inconclusive or not available. Both U07.1 and U07.2 may be used for mortality coding (cause of death).

The secondary was patients who had a hospital admissions diagnosis linked to any form of pneumonia, mainly ICD-10 Diagnosis J18.9. Box 1 below depicts the diagnosis distribution of different types of pneumonia. ICD-10 Diagnosis J18.9 accounted for nearly two-thirds of admissions. The last criterion was other types of admissions diagnosis. Predictors of readmissions were modeled using logistic regression. For this study, readmissions, as was defined, are readmissions within 30 days of the first admission.

\section{Statistical analysis}

Categorical variables were described by means of frequency counts and percentages. Continuous variables were smamrised and mean, median, and interquartile range (IQR) values reported. Differences between continuous variables was used by means of t-test and proportions between categorical variables were done using the $x 2$ test, although the Fisher exact test was used when the data were limited. Statistical analyses were performed using STATA 14 and SAS 9.4 (North Carolina, USA). The relationship between demographic characteristics, risk factors and hospitalisation were modelled using a logistic regression model.

\section{RESULTS}

\section{Demographic characteristics}

The analysis covered a total of 1159 close scheme patients who were admitted to private hospitals with a median age of 55 (IQR 47-65) years. Table 1 depicts the different demographic characteristics of COVID-19-confirmed diagnosis, 'J18.9 Pneumonia, unspecified organism' and other non-COVID-19 related admissions, stratified by risk factors such gender and age bands, outcomes (deaths), and admitting facilities. Females made up sixty two percent $(n=719)$ of study participants. Sixty-four percent $(n=360)$ of all confirmed COVID-19 diagnoses were females, while fifty-eight percent $(n=283)$ of non-COVID-19 diagnoses were females and sixty-six percent $(n=76)$ among 'J18.9 Pneumonia, unspecified organism' patients were females. Among COVID19 confirmed-patients, $51.8 \%$ were aged 55 years or older, compared to $46.3 \%$ and $45.2 \%$ nonCOVID-19 and 'J18.9 Pneumonia, unspecified organism' patients, respectively. Table 1 further depicts that $22 \%$ of Pneumonia-unspecified admissions potentially developed into COVID-19 diagnoses. Furthermore $12 \%$ of COVID-19 changed to Pneumonia cases. Other types of admissions that changed into either COVID-19 or Pneumonia accounted for 9 and 2 percent, respectively. Thus, 89 percent of admissions 


\section{ICD-10 Primary Admission Diagnosis Diagnosis Description}

\section{$\%$}

J18.9

Pneumonia, unspecified

$74 \%$

J18.0

Bronchopneumonia, unspecified

$18 \%$

J18.1

Lobar pneumonia, unspecified

$4 \%$

$\mathrm{J} 18.8$

Other pneumonia, organism unspecified

J18.2

Hypostatic pneumonia, unspecified

Box 1: Distributions of Pneumonia admissions diagnosis codes

Table 1. Demographic characteristics [n]

\begin{tabular}{|c|c|c|c|c|c|}
\hline Row Labels & $\begin{array}{l}\text { COVDI-19: } \\
\text { Emergency use of } \\
\text { U07.1 }\end{array}$ & Other & $\begin{array}{l}\text { ‘J18.9 Pneumonia, } \\
\text { unspecified } \\
\text { organism’ } \\
\text { unspecified }\end{array}$ & Grand Total & $\begin{array}{l}\text { Chi-square, } \\
\text { p-value }\end{array}$ \\
\hline \multicolumn{6}{|l|}{ Gender } \\
\hline Female & 360 & 283 & 76 & 719 & \multirow[t]{2}{*}{$x^{2}=5.2472, p=0.0725$} \\
\hline Male & 198 & 203 & 39 & 440 & \\
\hline \multicolumn{6}{|l|}{ Age groups } \\
\hline$<35$ years & 30 & 58 & 17 & 105 & \multirow{5}{*}{$\begin{array}{l}x^{2}=28.0067 \\
p=0.0142\end{array}$} \\
\hline $35-44$ years & 80 & 66 & 12 & 158 & \\
\hline $45-54$ years & 163 & 137 & 34 & 334 & \\
\hline $55-64$ years & 155 & 108 & 25 & 288 & \\
\hline $65+$ years & 130 & 117 & 27 & 274 & \\
\hline \multicolumn{6}{|l|}{ Admitting facility } \\
\hline General Ward & 139 & 258 & 60 & 457 & \multirow{4}{*}{$\begin{array}{l}x^{2}=187.6011 \\
p<.0001\end{array}$} \\
\hline High Care & 104 & 103 & 26 & 233 & \\
\hline ICU & 74 & 81 & 15 & 170 & \\
\hline $\begin{array}{l}\text { Other types of admitting } \\
\text { facilities. }\end{array}$ & 241 & 44 & 14 & 299 & \\
\hline \multicolumn{6}{|l|}{ Discharge status } \\
\hline Emergency use of U07.1 & 0 & 43 & 26 & 69 & \multirow{4}{*}{$\begin{array}{l}x^{2}=106.8735 \\
p<.0001\end{array}$} \\
\hline Other physical therapy & 0 & 1 & 0 & 1 & \\
\hline $\begin{array}{l}\text { 'J18.9 Pneumonia, } \\
\text { unspecified organism' } \\
\text { unspecified }\end{array}$ & 68 & 11 & 3 & 82 & \\
\hline Other types of diagnosis & 490 & 431 & 86 & 1007 & \\
\hline \multicolumn{6}{|l|}{ Outcomes } \\
\hline Deceased & 96 & 48 & 8 & 152 & \multirow{3}{*}{$\begin{array}{l}x^{2}=16.4897 \\
p=0.0003\end{array}$} \\
\hline Alive & 462 & 438 & 107 & 1007 & \\
\hline Grand Total & 558 & 486 & 115 & 1159 & \\
\hline
\end{tabular}


Table 2. Inpatient days and risk benefits paid [median (interquartile range)

\begin{tabular}{|c|c|c|c|}
\hline ICD Primary Admission Category & $\begin{array}{l}\text { Admission Type } \\
\text { Description }\end{array}$ & $\mathbf{N}^{\star \star}$ & Median (IQR) \\
\hline \multicolumn{4}{|l|}{ Emergency use of U07,1 } \\
\hline \multirow[t]{2}{*}{ General Ward } & Risk benefit paid (R) & \multirow[t]{2}{*}{129} & $28454(18262-48403)$ \\
\hline & Inpatient days & & $7(4-10)$ \\
\hline \multirow{2}{*}{ High Care } & Risk benefit paid (R) & \multirow[t]{2}{*}{64} & $97080(59770-153145)$ \\
\hline & Inpatient days & & $9(7-13)$ \\
\hline \multirow[t]{2}{*}{ ICU } & Risk benefit paid $(\mathrm{R})$ & \multirow[t]{2}{*}{10} & $194564(86970-229$ 144) \\
\hline & Inpatient days & & $13(11-16)$ \\
\hline \multirow[t]{2}{*}{ Other types of admitting facilities } & Risk benefit paid (R) & \multirow[t]{2}{*}{248} & 31108 (18 $388-62385)$ \\
\hline & Inpatient days & & $7(4-10)$ \\
\hline \multicolumn{4}{|l|}{ Other types of diagnosis } \\
\hline \multirow[t]{2}{*}{ General Ward } & Risk benefit paid $(\mathrm{R})$ & \multirow[t]{2}{*}{359} & $19900(11434-40540)$ \\
\hline & Inpatient days & & $4(3-7)$ \\
\hline \multirow[t]{2}{*}{ High Care } & Risk benefit paid (R) & \multirow[t]{2}{*}{92} & 42728 (21 $086-88$ 282) \\
\hline & Inpatient days & & $7(3-11,17)$ \\
\hline \multirow[t]{2}{*}{ ICU } & Risk benefit paid $(\mathrm{R})$ & \multirow[t]{2}{*}{49} & 82407 (46 $306-160628)$ \\
\hline & Inpatient days & & $8(6-13)$ \\
\hline \multirow[t]{2}{*}{ Other of admitting facilities } & Risk benefit paid $(\mathrm{R})$ & \multirow[t]{2}{*}{105} & $10847(1479-39414)$ \\
\hline & Inpatient days & & $6(4-11,5)$ \\
\hline \multicolumn{4}{|c|}{ 'J18.9 Pneumonia, unspecified organism' unspecified } \\
\hline \multirow[t]{2}{*}{ General Ward } & Risk benefit paid (R) & \multirow[t]{2}{*}{20} & $23288(13780-30845)$ \\
\hline & Inpatient days & & $6(4-8,5)$ \\
\hline \multirow[t]{2}{*}{ High Care } & Risk benefit paid (R) & \multirow[t]{2}{*}{12} & $53556(38004-130002)$ \\
\hline & Inpatient days & & $7(5-8)$ \\
\hline \multirow[t]{2}{*}{ ICU } & Risk benefit paid (R) & \multirow[t]{2}{*}{3} & $48029(20856-168485)$ \\
\hline & Inpatient days & & $6(3-18)$ \\
\hline \multirow[t]{2}{*}{ Other of admitting facilities } & Risk benefit paid (R) & \multirow[t]{2}{*}{14} & $17398(1677-34412)$ \\
\hline & Inpatient days & & $5(9,5-13)$ \\
\hline
\end{tabular}

changed into other diagnoses other than Pneumonia or COVID-19 at the discharge phase.

\section{Utilisation of services}

Table 2 depicts utilisation statistics of health facilities for the three admission categories. Extreme events where a risk-benefit was paid, and inpatient days were higher than the 90th percentile were excluded from the analysis. Patients admitted for COVID-19 were chiefly admitted in the "other" type of admitting facilities, compared to those admitted in the general ward, high care, and ICU. The admission rates were 55 percent, 29 percent, 14 percent and 2 percent, respectively. 'J18.9 Pneumonia, unspecified organism' admissions were chiefly in the general ward, compared to high care, ICU, and other types of admission.

The hospitalisation rates or the facility were 41 percent, 24 percent, 6 percent, and 29 percent, respectively. For ICU types of admissions, there were nearly three times more 'J18.9 Pneumonia, unspecified organism' admissions compared to COVID-19 admissions, 6 percent vs. 2 percent. However, COVID-19 ICU inpatients stayed longer 


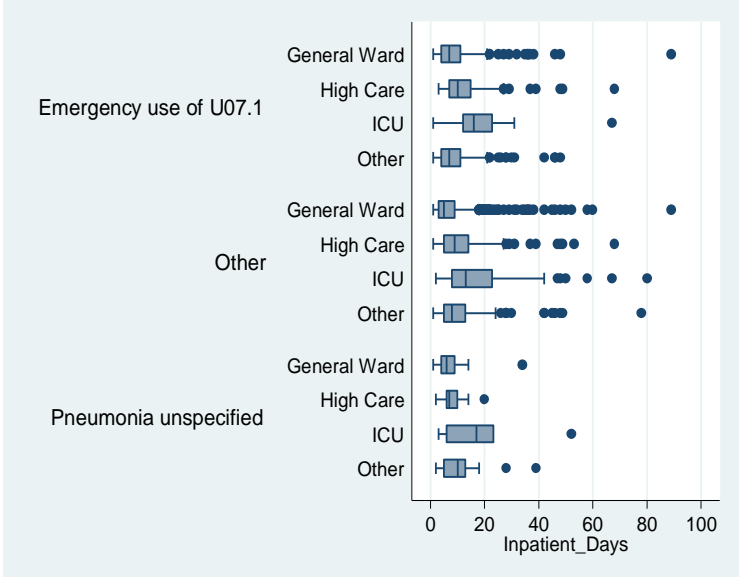

Figure 1. Inpatient days by primary admission ICD-10 diagnosis category and admitting (restricted to $90^{\text {th }}$ percentiles)

than 'J18.9 Pneumonia, unspecified organism' diagnosis, with median inpatient days of 13 and 6 days, respectively. A similar phenomenon was also observed in high care admissions, where the median inpatient days was 9 days vs. 7 days, respectively. The distribution spread in the expenditure per event was wider in an ICU setting with COVID-19 admissions at a 75th percentile higher than the other admission facilities, which was nearly R230 000. The second highest was for 'J18.9 Pneumonia, unspecified organism' admissions, which was nearly R670 000, another type of admitting facilities attracted 75th percentile expenditure per event of just over R160 000. It should be noted, however, that 'J18.9 Pneumonia, unspecified organism' admissions in an ICU setting were proportionally small, compared to the two groups.

Figures 1 and 2 show a box and whisker plot of inpatient days by admitting facility and risk benefits paid. Variability in the inpatient days and risk-benefit paid per event was more evident in COVD-19 admissions and Other types of admissions compared to 'J18.9 Pneumonia, unspecified organism' admissions.

\section{Admission trends}

Figure 3 depicts trend data in respect of the number of admissions of a select set of admission diagnoses. The results show an increase in

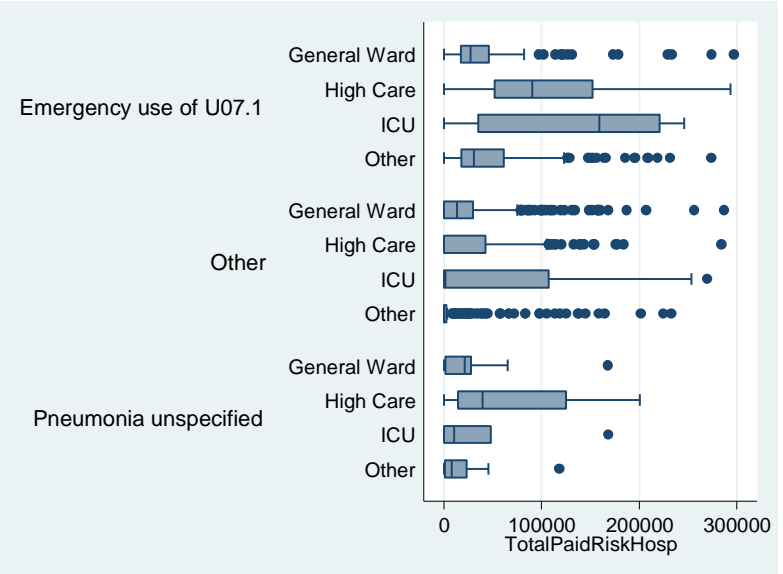

Figure 2. Risk benefits by primary admission ICD-10 diagnosis category and admitting facility type (restricted to $90^{\text {th }}$ percentiles)

admissions during June and the number of admissions picked in July. It was also evident from the data that some confirmed COVID-19 diagnoses were observed during June. This picked up in July where COVID-19 accounted for almost more than half of COVID-19 admissions. $50 \%$ and $47 \%$ of diabetes type $1 \& 2$ and 'J18.9 pneumonia, unspecified organism' admissions, respectively. During August, the figures declined for all three conditions. The increase observed in July is also consistent with the increase observed at the national level, like the downward trend noted in August.

\section{Primary admission and discharge diagnosis}

Table 3 and 4 below show the top 10 primary and discharge ICD-10 diagnosis codes for patients admitted for COVID-19, 'J18.9 Pneumonia, unspecified organism' and other conditions. Among the main reasons for primary admission were confirmed diagnosis or case of COVID-19 (emergency use of U07.1: confirmed COVID-19 cases or diagnosis). Emergency use of U07.1 (confirmed COVID-19 cases or diagnosis) was also the most prevalent primary discharge diagnosis in hospitals. This might be associated with patients who still had COVID-19 but were discharged to recover under home-based care. The other primary admission diagnoses included pneumonia - unspecified, Unspecified acute lower respiratory infection, 


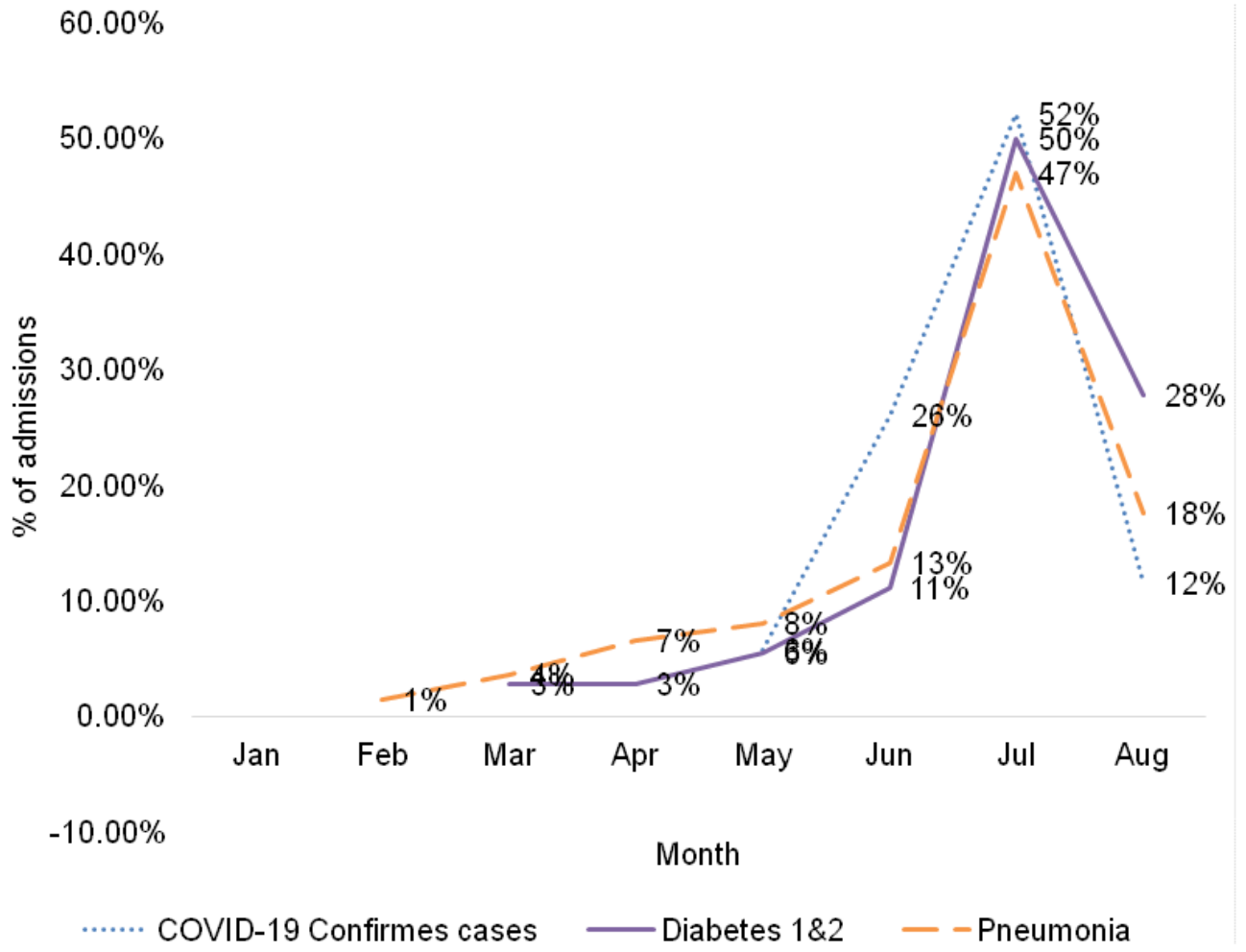

Figure 3. Number of admissions of 'J18.9 Pneumonia, unspecified organism' patients by month and admission diagnosis category

Table 3. Primary admission and discharge diagnosis - Top 10: counts

\begin{tabular}{|c|c|c|c|}
\hline \multicolumn{4}{|c|}{ ICD-10 Primary Admission Diagnosis } \\
\hline ICD-10 Code & ICD-10 Description & $\mathrm{N}$ & $\%$ \\
\hline U07.1 & Emergency use of U07.1 & 558 & 48 \\
\hline J18.9 & Pneumonia, unspecified & 115 & 10 \\
\hline J22 & Unspecified acute lower respiratory infection & 44 & 4 \\
\hline J12.8 & Other viral Pneumonia & 35 & 3 \\
\hline J80 & Adult respiratory distress syndrome & 22 & 2 \\
\hline J18.0 & Bronchopneumonia, unspecified & 21 & 2 \\
\hline A09.9 & Gastroenteritis and colitis of unspecified origin & 15 & 1 \\
\hline R06.0 & Dyspnoea & 13 & 1 \\
\hline U07.2 & Emergency use of U07.2 & 13 & 1 \\
\hline J96.99 & Respiratory failure, unspecified & 11 & 1 \\
\hline
\end{tabular}

Other viral 'J18.9 Pneumonia, unspecified organism' adult respiratory distress, syndrome, Broncho'J18.9 Pneumonia, unspecified organism' -unspecified.
Table 4 shows that Isolation, Pneumoniaunspecified, Emergency use of U07.1, Essential (primary) hypertension Dependence on a respirator, dyspnoea, and special screening 
Table 4. Primary discharge diagnosis - Top 10: counts

\begin{tabular}{|l|l|l|l|}
\hline ICD-10 Primary Discharge Diagnosis & N & $\%$ \\
\hline ICD-10 Code & ICD-10 Code description & 110 & 9.5 \\
\hline $\mathbf{Z 2 9 . 0}$ & Isolation & 82 & 7.1 \\
\hline $\mathbf{J 1 8 . 9}$ & Pneumonia, unspecified & 69 & 6.0 \\
\hline $\mathbf{U 0 7 . 1}$ & Emergency use of U07.1 & 64 & 5.5 \\
\hline $\mathbf{I 1 0}$ & Essential (primary) hypertension & 38 & 3.3 \\
\hline $\mathbf{Z 9 9 . 1}$ & Dependence on respirator & 33 & 2.8 \\
\hline $\mathbf{R 0 6 . 0}$ & Dyspnoea & 31 & 2.7 \\
\hline $\mathbf{Z 1 1 . 5}$ & Special screening examination for other viral diseases & 2.6 \\
\hline $\mathbf{U 0 7 . 2}$ & Emergency use of U07.2 & 30 & 2.5 \\
\hline $\mathbf{J} \mathbf{0 0}$ & Adult respiratory distress syndrome & 29 & 2.5 \\
\hline $\mathbf{Z 0 3 . 8}$ & Observation for other suspected diseases and conditions & 29 & \\
\hline
\end{tabular}

\begin{tabular}{|c|c|c|c|c|c|}
\hline \multirow[t]{7}{*}{ 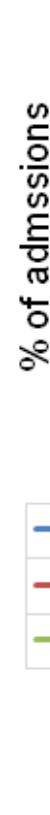 } & \multirow[t]{2}{*}{$\begin{array}{r}90.0 \% \\
80.0 \% \\
70.0 \% \\
60.0 \% \\
50.0 \% \\
40.0 \% \\
30.0 \% \\
20.0 \% \\
10.0 \% \\
0.0 \%\end{array}$} & \multirow[b]{2}{*}{1} & & & \multirow[b]{2}{*}{$3+$} \\
\hline & & & 2 & 3 & \\
\hline & of U07.1 & $77.3 \%$ & $19.9 \%$ & $1.8 \%$ & $1.0 \%$ \\
\hline & & $44.7 \%$ & $32.0 \%$ & $10.2 \%$ & $13.1 \%$ \\
\hline & pecified & $79.5 \%$ & $13.6 \%$ & $6.8 \%$ & \\
\hline & \multirow{2}{*}{\multicolumn{2}{|c|}{ cy use of U07.1 }} & & & \\
\hline & & & & \multicolumn{2}{|c|}{ Pneumonia unspecified } \\
\hline
\end{tabular}

Figure 4. Number of admissions by admission type categories identification category

examination for other viral diseases were among the most prevalent primary discharge diagnoses.

\section{Readmission rates by COVID-19 identification category}

Figure 4 below depicts the number of admissions per patient over the review period. Four groups were compared, namely, those who were admitted for COVID-19, Pneumonia, other types of diagnosis, and at the consolidated level. The three groups were statistically significant (MantelHaenszel Chi-square $=25.44, \quad \mathrm{p}<0.0001)$. Significantly more of the patients were admitted only once: 79.5 percent, 77.3 percent, and 44.7 percent for Pneumonia, COVID-19, and other types of admissions, respectively. More COVID19 diagnoses were admitted twice compared to those that were initially admitted for pneumonia, 


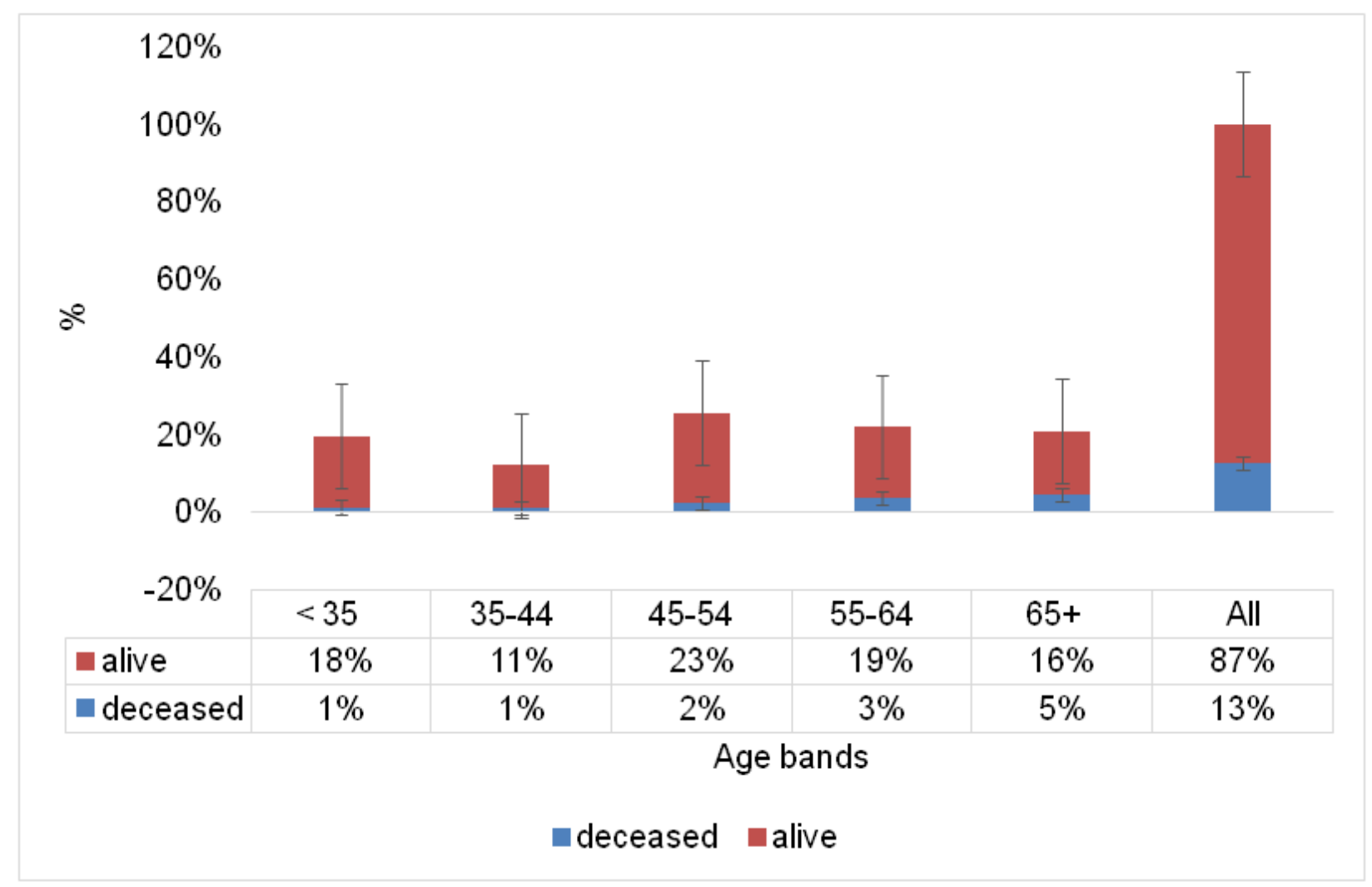

Figure 5. Number of deaths by age category

$19.9 \%$ and $13.6 \%$, respectively. This trend was, however, reversed when looking at patients that were admitted three times, $1,8 \%$ compared to $6.8 \%$, respectively.

\section{Mortality}

Figure 5 below shows a mortality rate of $13 \%$, and this was proportional to the older age bands where the age band $65+$ years accounted for $5 \%$ of deaths. The age band 45-54 years accounted for nearly a quarter of patients that survived.

\section{Multiple logistic regression model}

The outcome variable was the readmission rate of patients admitted to a private hospital facility. Independent variables included age (5 age bands), gender (Male vs. Female), admitting facility (general ward, high care, and ICU), and primary diagnosis at admission (confirmed COVID-19 diagnosis, 'J18.9 Pneumonia, unspecified organism' confirmed case and other types of cases.

Table 5 depicts the results of a logistics regression model. The results show that the primary diagnosis age and admitting facility were statistically significant to the outcome variable. Patients in the age band < 35 years were associated with a decreased risk of readmission, compared to age band 45-54 years and 55-64 years (OR=0.659 Cl: $0.392-1.108, p=0.0393$ ) which was statistically significant and $(\mathrm{OR}=0.686$ $\mathrm{Cl}$ : $0.0 .402-1.17, p=0.094)$ which was marginally significant, respectively. Female patients were associated with an increased risk of readmission compared to male patients (OR=3.44 Cl: 2.478 $4.779, p<0.001)$. Lastly, the ICU admission facility 
Table 5: Logistic regression: Outcome variable readmission rates

\begin{tabular}{|c|c|c|c|c|}
\hline & & OR & $\begin{array}{l}95 \% \text { Confidence } \\
\text { Interval }\end{array}$ & $\mathbf{P}$ \\
\hline Gender category Ref (Female) & Male & 1.099 & $(0.82-1.462)$ & 0.5367 \\
\hline \multirow{2}{*}{$\begin{array}{l}\text { Primary Admission Diagnosis } \\
\text { Category (Ref COVID-19 } \\
\text { positive) }\end{array}$} & Other & 3.441 & $(2.478-4.779)$ & $<.0001$ \\
\hline & $\begin{array}{l}\text { Pneumonia, } \\
\text { unspecified }\end{array}$ & 1.179 & $(0.676-2.057)$ & 0.084 \\
\hline \multirow[t]{2}{*}{ Age category } & $35-44$ years & 1.036 & $(0.584-1.84)$ & 0.2873 \\
\hline & $45-54$ years & 0.659 & $(0.392-1.108)$ & 0.0393 \\
\hline \multirow[t]{2}{*}{ Ref (< 35 years) } & $55-64$ years & 0.686 & $(0.402-1.17)$ & 0.0944 \\
\hline & $65+$ years & 1.057 & $(0.628-1.78)$ & 0.1427 \\
\hline \multirow{3}{*}{$\begin{array}{l}\text { Admission facility type } \\
\text { Ref (ICU) }\end{array}$} & General Ward & 0.57 & $(0.381-0.852)$ & 0.1816 \\
\hline & High Care & 0.797 & $(0.511-1.245)$ & 0.1708 \\
\hline & Other & 0.430 & $(0.263-0.703)$ & 0.0046 \\
\hline
\end{tabular}

was associated with a decreased risk of readmissions compared to another type of admitting facilities $(\mathrm{OR}=0.430 \mathrm{Cl} 0.263-0.703$, $p=0.005)$; this was statistically significant.

\section{DISCUSSION}

This study sought to assess the characteristics of patients admitted to the hospital for COVID-19, 'J18.9, Pneumonia, unspecified organism' and other types of diagnosis. This study found that the median age of pneumonia patients admitted to the hospital for COVID-19 was 55 (IQR 47-65) years. This demographic of patients was consistent with a study conducted by Chen et al. (2020), who studied the epidemiological and clinical characteristics of 2019-nCoV pneumonia. Their study found that the average age of the patients was 55.5 years (SD 13.1), including 67 men and 32 women. Our study found that significantly more of admitted pneumonia patients were females, which consisted of nearly two-thirds of admissions. COVID-19 is generally characterised with a higher effect on older patients. Furthermore, demographic characteristics such as age and gender have been found to increase the risk of COVID-19, mainly in older patients. A study by Jin et al. (2020) revealed that older age and a higher number of comorbidities were associated with greater severity and mortality in patients with both COVID-19 and SARS. Among COVID-19 confirmed-patients, $51.8 \%$ were aged 55 years or older, compared to $46.3 \%$ and $45.2 \%$ non-COVID19 and 'J18.9 Pneumonia, unspecified organism' patients, respectively. These findings are in contrast with a study done by Garcia-Vidal et al. (2020), who assessed 989 consecutive adults with COVID-19 who either had been discharged or died during the study period. Their study found that more of the patients were males $552(55.8 \%)$ and were slightly older than 60 years; the median (IQR) age was 62 years.

The study found that 22 percent of Pneumoniaunspecified admissions changed into a COVID-19 diagnosis. Studies have, however, depicted that pneumonia is a complication from COVID-19. The COVID-19 is caused by severe acute respiratory syndrome coronavirus 2 (SARS-CoV-2) virus that causes pneumonia; however, in more severe cases, it affects other organs (Jager et al., 2020). According to the Centre for Disease Control and Prevention (CDC), in the United States, the common causes of viral 'J18.9 Pneumonia, unspecified organism' are influenza, respiratory syncytial virus (RSV), and SARS-CoV-2 (the virus that causes COVID-19). Our study found that indeed pneumonia could change to a COVID-19 diagnosis, thus emphasising the reliance and reliability in the primary admissions and primary discharge diagnosis, which is more reliable. 
Our study found that only 12 percent of COVID19 diagnosis changed into a Pneumonia diagnosis. This is consistent with the literature in that COVID-19 patients can present as asymptomatic, mild upper respiratory tract disease, or potentially severe pneumonia (Bruminhent et al., 2020). According to Zhu et al. (2020), SARS-CoV-2 is an emerging respiratory virus that commonly causes tract infection and is occasionally complicates to severe pneumonia. Bruminhent et al. (2020) classified nearly seventy percent COVID-19 patients as having upper respiratory tract infection, just over fifteen percent as having a viral syndrome (15.1\%), and just over ten percent as having pneumonia (11.3\%), or asymptomatic infection (3.8\%). The main finding and key insights from our study are that the reverse is also true in that pneumonia could complicate or changed to a COVID-19 diagnosis. Thus, depicting pneumonia as a potential risk factor associated with COVID-19. Clinical characteristics of COVID-19 related pneumonia patients have been shown in recent studies (Wang et al., 2020; Chen et al., 2020; Zhu et al., 2020). A few studies have also indicated instances of cases where COVID-19 causes pneumonia and fatal respiratory distress (WHO, 2020). Other types of admissions that changed to either COVID-19 or pneumonia accounted for 9 and $2 \%$, respectively. This study further depicted a close relationship between pneumonia and COVID-19 in terms of health care utilisation rates. The study found a similar risk of admissions where the rate readmissions were at least twice was just over a fifth, $22.6 \%$ for COVID-19 readmissions and $20.4 \%$ pneumonia readmissions, respectively. This further depicts similar characteristics between pneumonia and COVID-19.

COVID-19 ICU inpatients stayed longer than 'J18.9 Pneumonia patients, unspecified organism' diagnoses, with median inpatient days of 13 and 6 days, respectively. According to the report by the American Journal of Managed Care, patients who are admitted to hospital for pneumonia, intensive care unit stay increased time spent in the hospital by half, resulting to a length of stay to 7.2 days (Bai, 2018). Patients admitted for COVID-19 were chiefly admitted in the "other" type of admitting facilities compared to those admitted in the general ward, high care, and an intensive care unit. The respective admission rates were, $55 \%$,
$29 \%, 14 \%$ and $2 \%$ respectively. A study by Bruminhent et al. (2020) depicted a similar trend in so far as intensive care admissions are concerned, which accounted for $1.9 \%$, the remaining $98.1 \%$ were admitted in the general ward.

'J18.9 Pneumonia, unspecified organism' admissions were chiefly in the general ward, compared to high care, intensive care, and other types of admission. The hospitalisation rates by admitting facility type were $41 \%, 24 \%, 6 \%$, and $29 \%$, respectively. For intensive care types of admissions, there were nearly three times more 'J18.9 Pneumonia, unspecified organism' admissions compared to COVID-19 admissions, $6 \%$ vs. $2 \%$. However, COVID-19 ICU inpatient stay was longer than 'J18.9 Pneumonia, unspecified organism' diagnosis, with median inpatient days of 13 and 6 days, respectively.

Our study shows a similar length of stay in ICU compared to China, which is higher than in countries outside China. ICU length of stay was reported by eight studies-four each within and outside China-with median values range from 6 to 12 and 4 to 19 days, respectively (Eleanor et al., 2020). The median lengths of stay in the hospital for COVID-19-related admissions and other types of admissions were similar at just under eight days, as were the median costs per event. The study found that some pneumonia patients were subjected to co-payments as, in some instances, the medical scheme did not cover the whole amount claimed. This was slightly significantly lower in COVID-19-related admissions. COVID-19 has been declared as a PMB condition that schemes need to cover the treatment cost (CMS, 2020). Similarly, Pneumonia patients are also a PMB condition. It is therefore worrying that some members were subjected to co-payments as high as $7 \%$ of the amount claimed.

The logistic regression results showed that primary diagnosis age and admitting facility were statistically significant to the outcome variablereadmissions. The study found that patients in the age band $<35$ years were associated with a decreased risk of readmission. Female patients were associated with an increased risk of readmission compared to male patients; the ICU admission facility was associated with a decreased risk of readmissions compared to another type of admitting facilities. These findings 
were consistent with the literature; a study by Donnelly et al. (2020) showed that within two months of discharge, 354 patients (19.9\%) who survived COVID-19 hospitalisation were readmitted. The most common readmission diagnoses were COVID-19 (30.2\%), sepsis $(8.5 \%)$, pneumonia $(3.1 \%)$, and heart failure (3.1\%).

Himmels et al. (2020) assessed the risk factors for ICU admissions due to COVID-19, but information on ICU admission was only provided by a single study with descriptive information. Age was found to be the strongest predictor of severe COVID-19, with a dose-response relationship between increasing age and increased risk of ICU admission. Moreover, male sex, morbid obesity (BMl>40), heart failure, and diabetes were associated with three increased risks for severe COVID-19 (Himmels et al., 2020).

In addition to a COVID-19 and Pneumonia related primary diagnosis, the study found that dyspnoea was one of the symptoms of admission. Dyspnoea which is also known as shortness of breath or breathlessness, is a subjective awareness of the sensation of uncomfortable breathing. A study by Leitmeyer et al. (2020) showed that a dry cough, chest pain and dyspnoea were prominent in patients with lower respiratory tract symptoms on admission. The other diagnosis for admission were Unspecified acute lower respiratory infection, Other viral Pneumonia, Adult respiratory distress syndrome, Bronchopneumonia, unspecified - Gastroenteritis, and colitis of unspecified origin. The study found that Pneumonia patients admitted to the hospital were susceptible to other chronic conditions such as essential (primary) hypertension.

\section{CONCLUSION}

This study revealed evidence that patients admitted for other types of diagnosis changed into a COVID-19 or Pneumonia diagnosis. The risk was, however, higher in changing to a COVID-19 type of diagnosis at discharge. It is concerning that some COVID-19 patients are readmitted more than once, and lastly, that risk factors such as age and gender were found to be predictors of readmissions. The study found that restricted scheme patients admitted for COVID-19 have the potential of changing to 'J18.9 Pneumonia, unspecified organism' diagnosis.
The study also showed some evidence that patients who were admitted for 'J18.9 Pneumonia, unspecified organism' changed to a COVID-19 diagnosis. The study also revealed evidence that patients admitted for other types of diagnosis changed to a COVID-19 or Pneumonia diagnosis. The risk was however higher in changing to a COVID-19 diagnosis at discharge stage. Risk factors such as age and gender were found to be predictors of readmissions. One of the main key findings of this study was evidence that COVID-19 patients were readmitted more than once, thus depicting, heightened risk of clinical deterioration on these patients. Thus, indicating a need for continued care post first admission.

\section{Limitations and future research}

The study did not conduct a comprehensive analysis of condition-specific readmissions, furthermore, the study did not consider severity and comorbidities among patients that were admitted in hospitals, these key attributes could certainly enhance the findings of this study. An industry-wide study could certainly provide better insight at the industry level could assist policymakers to make an informed decision on key interventions on COVID-19, pneumoniarelated admissions. Future research should entail detailed analysis and audit of discharge diagnosis, including those that are related to mortality.

\section{Competing interests}

The authors have declared that no competing interest exists.

\section{Authors' contributions}

Both authors contributed equally to this work.

\section{REFERENCES}

Audley G, Frankenfeld P, Dlamini S, Raubenheimer P, Hoare J, Cairncross L, Berg R, Gule M, Calligaro G (2020). Prolonged Treatment of COVID-19 'J18.9 Pneumonia, unspecified organism' With High-Flow Nasal Oxygen: A Story of Oxygen and Resilience. [Online] Samj.org.za. Available <http://samj.org.za/index.php/samj/article/view/13112> [Accessed 6 November 2020]. 
Bai D (2018). Intensive Care Unit Usage for 'J18.9 Pneumonia, unspecified organism' Doubles Length of Hospital Stay. [Online] AJMC. Available at: $<$ https://www.ajmc.com/view/intensive-care-unit-usage-forpneumonia-doubles-length-of-hospital-stay> [Accessed 6 November 2020].

Berber S, Reed L, Syam N, Jones N (2020). Severe mental illness and risks from COVID-19. Available at https://www.cebm.net/covid-19/severe-mental-illness-andrisks-from-covid-19/

Berenguer J, Ryan P, Rodríguez-Baño J (2020). Characteristics and predictors of death among 4,035 consecutively hospitalized patients with COVID-19 in Spain. Clin. Microbiol. Infect. 26:1525-1536. Published online August 26 [Accessed 28 January 2021].

Biagi L, Rossi A, Malagoli A (2020). Clinical and epidemiological characteristics of 320 deceased Covid-19 patients in an Italian province: a retrospective observational study. J. Med. Virol. https://doi.org/10.1002/jmv.26147

Bruminhent J, Ruangsubvilai N, Nabhindhakara J, Ingsathit A, Kiertiburanakul S (2020). Clinical characteristics and risk factors for coronavirus disease 2019 (COVID-19) among patients under investigation in Thailand. PLoS ONE 15(9): e0239250. https://doi.org/10.1371/journal.pone.0239250

Cummings MJ, Baldwin MR, Abrams D, (2020). Epidemiology, clinical course, and outcomes of critically ill adults with COVID-19 in New York City: a prospective cohort study. Lancet. 395: 1763-1770.

Bornstein SR, Dalan R, Hopkins D, Mingrone G, Boehm BO (2020). Endocrine and metabolic link to coronavirus infection. Nat. Rev. Endocrinol. 16(6): 297-298. https://doi.org/10.1038/s41574-02

Centre for Disease Control (CDC). (2020). Causes of 'J18.9 Pneumonia, unspecified organism'| CDC. [Online] Available at: $\quad<$ https://www.cdc.gov/pneumonia/causes.html> [Accessed 5 November 2020].

Chen N, Zhou M, Dong X (2020). Epidemiological and clinical characteristics of 99 cases of 2019 novel coronavirus 'J18.9 Pneumonia, unspecified organism' in Wuhan, China: a descriptive study. Lancet. 395(10223), pp.507-513.

Cook TM (2020). The importance of hypertension as a risk factor for severe illness and mortality in COVID-19. Anaesthesia; 75(7): 976-977. https://doi.org/ 10.1111/anae.15103.

Council for Medical Schemes (2019). Draft PMB definition guidelines for mental health emergencies. Pretoria: Available at

https://www.medicalschemes.com/files/PMB\%20Definition\%20 Project/DraftPMBDefGuideline MentalHealthEmergencies 20190319.pdf

Council for Medical Schemes. (2020). PMB definition guideline: COVID-19 v2 05 May 2020. Pretoria: CMS. https://www.medicalschemes.com/files/PMB\%20Benefit\%20 definition\%20guidelines/PMB definition guideline-COVID19 v2 6 May 2020.pdf

Council for Medical Schemes. (2013). Circular 10 of 2013. Funding of PMBs from personal medical savings accounts when members are discharged from hospital. Council for Medical Schemes. Pretoria. South Africa. Available at: https://www.medicalschemes.com/files/Circulars/Circular10 Of2013.pdf

Cummings MJ, Baldwin MR, Abrams D (2020). Epidemiology, clinical course, and outcomes of critically ill adults with COVID-19 in New York City: a prospective cohort study. Lancet 2020. 395: 1763-1770.
Donnelly JP, Wang XQ, Iwashyna TJ, Prescott HC (2020). Readmission and Death After Initial Hospital Discharge Among Patients With COVID-19 in a Large Multihospital System. JAMA. JAMA 325:304-306

Fujita J (2020). SARS-CoV-2 as a causative agent of idiopathic interstitial 'J18.9 Pneumonia, unspecified organism' and interstitial 'J18.9 Pneumonia, unspecified organism' associated with collagen vascular disorders. Respiratory Investigat. 58(6): pp.427-429.

Fang L, Karakiulakis G, Roth M (2020). Are patients with hypertension and diabetes mellitus at increased risk for COVID-19 infection? Lancet. Respir. Med; [published online ahead of print]. doi: 10.1016/S2213-2600(20)30116-8.

Fuller RL, Atkinson G, McCullough EC, Hughes JS (2013). Hospital readmission rates: the impacts of age, payer, and mental health diagnoses. J. Ambulatory. Care. Manage. 36(2): 147-55

Guiot J, Henket M, Frix A (2020). Single-center experience of patients with interstitial lung diseases during the early days of the COVID-19 pandemic. Respir. Investigat. 58(6): 437439.

Gupta R, Hussain A, Misra A (2020). Diabetes and COVID-19: evidence, current status and unanswered research questions. Eur. J. Clin. Nutr. 74: 864-870. https://doi.org/10.1038/s41430-020-0652-1.

Himmels JPW, Borge TC, Brurberg KG, Gravningen KM, Feruglio SL, Berild JD (2020). COVID-19: COVID-19 and risk factors for hospital admission, severe disease and death: Norwegian Institute of Public Health.

Huang C, Wang Y, Li X (2020). Clinical features of patients infected with 2019 novel coronavirus in Wuhan, China. Lancet. 395: 497-506.

Jager K, Kramer A, Chesnaye N (2020). Results from the ERA-EDTA Registry indicate a high mortality due to COVID19 in dialysis patients and kidney transplant recipients across Europe. Kidney Intl. 98:1540-8 10

Jin JM, Bai P, He W (2020). Gender differences in patients with COVID-19: Focus on severity and mortality. MedRxiv. doi: https://doi.org/10.1101/2020.02.23.20026864. Available at: https://www.frontiersin.org/articles/10.3389/fpubh.2020.0015 2/full> [Accessed 6 November 2020].

Meng Y, Wu P, Lu W (2020). Sex-specific clinical characteristics and prognosis of coronavirus disease-19 infection in Wuhan, China: a retrospective study of 168 severe patients PLoS Pathog. 16: 1-13.

Okusaga O, Yolken RH, Langenberg $P$ (2011). Association of seropositivity for influenza and coronaviruses with history of mood disorders and suicide attempts. J. Affect. Disord. 130: 220-225.

Rokadiya S, Gil E, Stubbs C, Bell D, Herbert R (2020). COVID19: Outcomes of patients with confirmed COVID-19 readmitted to hospital. J.Infect. 81(3): e18-e19. https://doi.org/10.1016/j.jinf.2020.07.007

Severance EG, Dickerson FB, Viscidi RP (2011). Coronavirus immunoreactivity in individuals with a recent onset of psychotic symptoms Schizophr. Bull. 37(1): 101-107.

Tabata S, Imai K, Kawano S (2020). Clinical characteristics of COVID-19 in 104 people with SARS-CoV-2 infection on the Diamond Princess cruise ship: A retrospective analysis. Lancet. Infect. Dis. 20: 1043-1050.

Van Rheenen TE, Meyer D, Neill E (2020). Mental health status of individuals with a mood-disorder during the COVID-19 pandemic in Australia: Initial results from the 
COLLATE project. J. Affect. Disord. 275: 69-77. Advance online publication. https://doi.org/10.1016/j.jad.2020.06.037

Vu HT, Leitmeyer KC, Le DH (2004). Clinical description of a completed outbreak of SARS in Vietnam, February-May 2003. Emerg. Infect. Diseases. 10(2): 334-338. https://doi.org/10.3201/eid1002.030761

WHO (World Health Organisation). (2020). Media Statement: Knowing the Risks for COVID-19. Geneva. [Online] Available at:<https://www.who.int/indonesia/news/detail/0803-2020-knowing-the-risk-for-covid-19> [Accessed 6 November 2020].

WHO. (2020). INTERNATIONAL GUIDELINES FOR CERTIFICATION AND CLASSIFICATION (CODING) OF COVID-19 AS CAUSE OF DEATH. World Health Organisation. Geneva. Available at : https://www.who.int/classifications/icd/Guidelines Cause of Death COVID-19.pdf

WHO. (2020). Emergency use ICD codes for COVID-19 disease outbreak. Geneva. Available at: https://www.who.int/classifications/icd/covid19/en/

Willie MM (2020) The Utilisation of Psychiatry and Other Mental Health Benefits by Members of Medical Schemes, South Africa. Med Clin Rev. Vol. 6 No. 5: 106

Wu P, Fang Y, Guan Z (2009). The psychological impact of the SARS epidemic on hospital employees in China: exposure, risk perception, and altruistic acceptance of risk. Can. J. Psychiatry. 54: 302-311.

Wu Z, McGoogan JM (2020). Characteristics of and Important Lessons from the Coronavirus Disease 2019 (COVID-19) Outbreak in China: Summary of a Report of 72314 Cases from the Chinese Center for Disease Control and Prevention. JAMA.

doi:10.1001/jama.2020.2648
Yang J, Zheng Y, Gou X (2020). Prevalence of comorbidities in the novel Wuhan coronavirus (COVID-19) infection: A systematic review and meta-analysis. Intl. J. Infect. Dis. 94: 91-95.

Zhang JJ, Dong X, Cao YY (2020). Clinical characteristics of 140 patients infected by SARS-CoV-2 in Wuhan, China. Allergy. [published online Feb 19]. doi:10.1111/all.14238

Zhu N, Zhang D, Wang W, Li X, Yang B, Song J (2020) A Novel Coronavirus from Patients with Pneumonia in China, 2019. N Engl J Med. 2020;382(8):727-33. Epub 2020/01/25. pmid:31978945.

\section{Endnotes}

'The Act that governs medica schemes in South Africa ii A restricted medical scheme is one does not open to the public for membership: it is often restricted to groups of people such as employees of a particular company or industry. These schemes are subject to the same regulations as open schemes, and also are governed by the Medical Schemes Act of 1998. 